\title{
ON THE DENSENESS OF MINIMUM ATTAINING OPERATORS
}

\author{
S. H. KulKaRni AND G. RAMESH
}

Abstract. Let $H_{1}, H_{2}$ be complex Hilbert spaces and $T$ be a densely defined closed linear operator (not necessarily bounded). It is proved that for each $\varepsilon>0$, there exists a bounded operator $S$ with $\|S\| \leqslant \varepsilon$ such that $T+S$ is minimum attaining. Further, if $T$ is bounded below, that is if there exists $m>0$ such that $\|T x\| \geqslant m\|x\|$ for every $x$ in the domain of $T$, then $S$ can be chosen to be rank one.

Mathematics subject classification (2010): 47A05, 47A55.

Keywords and phrases: Densely defined closed operator, minimum attaining operator, gap metric, Weyl's theorem, Lindenstrauss theorem.

\section{REFERENCES}

[1] Y. A. Abramovich and C. D. Aliprantis, An invitation to operator theory, Graduate Studies in Mathematics 50, American Mathematical Society, Providence, RI, 2002, MR 1921782.

[2] N. I. AkhiEZer And I. M. Glazman, Theory of linear operators in Hilbert space, Dover Publications, Inc., New York, 1993, Translated from the Russian and with a preface by Merlynd Nestell, Reprint of the 1961 and 1963 translations, Two volumes bound as one, MR 1255973.

[3] Adi Ben-Israel and Thomas N. E. Greville, Generalized inverses: theory and applications, Wiley-Interscience [John Wiley \& Sons], New York-London-Sydney, 1974, Pure and Applied Mathematics, MR 0396607.

[4] M. Sh. Birman and M. Z. Solomjak,, Spectral theory of selfadjoint operators in Hilbert space, Mathematics and its Applications (Soviet Series), Translated from the 1980 Russian original by S. Khrushchëv and V. Peller, D. Reidel Publishing Co., Dordrecht, 1987, MR 1192782.

[5] Xavier Carvajal and Wladimir Neves, Operators that attain their minima, Bull. Braz. Math. Soc. (N.S.) 45 (2014), no. 2, 293-312, MR 3249529.

[6] Per Enflo, Janice Kover and Laura Smithies, Denseness for norm attaining operator-valued functions, Linear Algebra Appl. 338 (2001), 139-144, MR 1861118.

[7] H. A. GINDLER AND A. E. TAYLOR, The minimum modulus of a linear operator and its use in spectral theory, Studia Math., Polska Akademia Nauk. Instytut Matematyczny. Studia Mathematica 22, MR 0151850.

[8] Simone GRAMSCH AND EBERHARD SCHOCK, Ill-posed equations with transformed argument, $\mathrm{Ab}$ str. Appl. Anal. (2003), no. 13, 785-791, MR 1996924.

[9] C. W. Groetsch, Spectral methods for linear inverse problems with unbounded operators, J. Approx. Theory 70 (1992), no. 1, 16-28, MR 1168372.

[10] RoBert C. JAMES, Reflexivity and the supremum of linear functionals, Ann. of Math. (2) 66 (1957), 159-169, MR 0090019.

[11] Robert C. James, Reflexivity and the sup of linear functionals, Proceedings of the International Symposium on Partial Differential Equations and the Geometry of Normed Linear Spaces (Jerusalem, 1972), vol. 13, 1972, pp. 289-300 (1973), MR 0338742.

[12] S. H. KUlKARNI AND G. RAMESH, A formula for gap between two closed operators, Linear Algebra Appl. 432 (2010), no. 11, 3012-3017, MR 2639265.

[13] S. H. Kulkarni And G. Ramesh, Absolutely minimum attaining closed operators, Preprint, http://arxiv.org/abs/1606.05736 (2016).

[14] JoRAm Lindenstrauss, On operators which attain their norm, Israel J. Math. 1 (1963), 139-148, MR 0160094. 
[15] Ritsuo Nakamoto, Gap formulas of operators and their applications, Math. Japon. 42 (1995), no. 2, 219-232, MR 1356379.

[16] Gert K. Pedersen, Analysis now, Graduate Texts in Mathematics, vol. 118, Springer-Verlag, New York, 1989, MR 971256.

[17] G. RAMESH, McIntosh formula for the gap between regular operators, Banach J. Math. Anal. 7, 2013, 97-106, MR 3004269.

[18] Michael Reed And Barry Simon, Methods of modern mathematical physics. I, second ed., Academic Press, Inc. [Harcourt Brace Jovanovich, Publishers], New York, 1980, Functional analysis, MR 751959.

[19] Walter Rudin,, Functional analysis, second ed., International Series in Pure and Applied Mathematics, McGraw-Hill, Inc., New York, 1991, MR 1157815.

[20] KonRAD SCHMÜDGEN, Unbounded self-adjoint operators on Hilbert space, Graduate Texts in Mathematics, vol. 265, Springer, Dordrecht, 2012, MR 2953553. 